\title{
Stress propagation through frictionless granular material
}

\author{
Alexei V. Tkachenko and Thomas A. Witten \\ The James Franck Institute, \\ The University of Chicago, Chicago, Illinois 60637 \\ (submitted to Physical Review E; typeset August 8, 2018)
}

\begin{abstract}
We examine the network of forces to be expected in a static assembly of hard, frictionless spherical beads of random sizes, such as a colloidal glass. Such an assembly is minimally connected: the ratio of constraint equations to contact forces approaches unity for a large assembly. However, the bead positions in a finite subregion of the assembly are underdetermined. Thus to maintain equilibrium, half of the exterior contact forces are determined by the other half. We argue that the transmission of force may be regarded as unidirectional, in contrast to the transmission of force in an elastic material. Specializing to sequentially deposited beads, we show that forces on a given buried bead can be uniquely specified in terms of forces involving more recently added beads. We derive equations for the transmission of stress averaged over scales much larger than a single bead. This derivation requires the Ansatz that statistical fluctuations of the forces are independent of fluctuations of the contact geometry. Under this Ansatz, the $d(d+1) / 2$-component stress field can be expressed in terms of a $d$-component vector field. The procedure may be generalized to non-sequential packings. In two dimensions, the stress propagates according to a wave equation, as postulated in recent work elsewhere. We demonstrate similar wave-like propagation in higher dimensions, assuming that the packing geometry has uniaxial symmetry. In macroscopic granular materials we argue that our approach may be useful even though grains have friction and are not packed sequentially.

PACS numbers: 46.10. $+\mathrm{z}$, 83.70.Fn
\end{abstract}

\section{INTRODUCTION}

The nature of the forces within a static pile of grains has proven more subtle than one might expect. Such a pile is an assembly of many hard, spheroidal bodies that maintain their positions via a balance of gravitational forces and contact forces with their neighbors [1,2]. On the one hand, determining these forces is a prosaic equilibrium problem. Since the number of grains is large, the long-established notions of continuum solid mechanics appear applicable. On the other hand, a pile of grains or beads is not a solid. The forces between beads are more problematic than those between the atoms of a conventional solid. These latter forces are smoothly varying on the scale of the separation and they arise from a potential energy that includes attraction. The forces on a grain are different. First, they vary sharply with interparticle distance, and there is no attraction. Second, the frictional part of a contact force is not determined by the macroscopic positions of the grains. Rather, it de- pends on the how each contact was formed. The resulting macroscopic behavior of the pile is also clearly different from that of a conventional solid. Arbitrarily slight forces can disturb the pile, so that the notion of stable equilibrium is suspect. Despite these complexities, we expect the mechanics of a granular pile to be universal. Hard, round grains appear to form piles of the same nature independent of their composition or detailed shape. We are led to think of these as nondeformable objects that exert normal forces of constraint and transverse forces limited by Coulomb's static friction limit.

Recently, a puzzling discovery has underlined the subtlety of the forces in a conical heap of poured sand [3. The supporting force under the center, where the pile is deepest, is not maximal. Instead, the maximal force occurs along a circle lying between the edge and the center of the pile. From this circle the force decreases to a minimum at the center. In order to explain this puzzling "central dip", a number of inventive approaches have been taken. Some [1] seek to account for the minimum qualitatively by viewing the pile as a stack of concentric "wigwams", whose sloping sides support the load. Others [5] have shown that the central dip is compatible with the conventional continuum mechanics, in which the pile is viewed as a central elastic zone flanked by an outer plastic zone which is at the Coulomb static friction limit. A third group 6, 14,15 has argued that granular material requires a new constitutive law, a homogeneous, local, linear constraint on the stress arising from the packing geometry. We shall call it a "null stress" law. Their proposed law gives a continuum mechanics as simple as that of a liquid or a solid, yet different from either. The hallmark of this law is the hyperbolic equations governing the transmission of forces. Hyperbolic equations, such as the wave equation, obey causality. The wave at the present is unaffected by the future. In the null-stress picture, the vertical direction plays the role of time. Accordingly, forces at a given point in the pile are only influenced by forces above that point. Force propagates as in a traveling wave. The transmission is unidirectional, in contrast with conventional continuum elasticity. The equations of continuum mechanics are elliptical. According to these, a contact within the pile should be influenced by all the forces above or below it, as sketched in Figure 1.

A separate approach has given indirect evidence for the unidirectional transmission of forces. Coppersmith's 17] heuristic $q$ model aims to account for the point-to-point variability of the contact forces. It imposes a unidirectional prescription for determining the downward forces 
from one grain in terms of the forces acting on it from above. Both this model and refinements 18, 19 of it yield an exponential falloff of probability for large forces. This exponential falloff agrees well with measured distributions [17,20,21]. This exponential falloff contrasts with the Gaussian falloff expected for a heterogeneous elastic solid.

In this paper we grapple with the relationship between the conventional elastic view and the newer null-stress picture. Our work builds on several recent studies of the relationship between the connectivity of a structure and the force transmission in it. Alexander's recent review [22] has explored the nature of unconstrained degrees of freedom in a minimally connected or isostatic [1] network. Ball and Edwards [11] have explored force transmission through minimally-connected networks, assuming a fixed co-ordination number for all particles. They have shown that such lattices can have constitutive equations of the null-stress form. Our main aim here is to broaden the class of systems that must show unidirectional force transmission, as required by the null-stress picture.

We present the discussion of the problem of stress transmission in a granular packing on several levels of generality. In sections $\mathbb{1}$ IV we focus on properties of the system of frictionless, spherical beads. Possible experimental realizations are hard-sphere colloidal dispersions 24,25. or weakly-deformed droplet emulsions [26]. First, in Section II we use general counting arguments to show that such a packing is minimally coupled. We then relate this fact to the inadequacy of elastic description for such a system. For a small subsystem within the pile a counting of equations and unknowns shows that approximately half of the surface forces transmitted from outside the subsystem are redundant. In equilibrium these cannot be independently specified, but have a fixed relation to the other half of the forces. This requirement for balance amongst many forces implies the existence of soft modes - infinitesimal deformations with no restoring force. In the continuum limit, the soft modes impose conditions on the stress field of the null-stress form.

In order to obtain the particular form of such macroscopic description, we limit further discussion to so-called sequential packing specified in Section III. In Section IV we give a microscopic prescription for determining the contact forces in a unidirectional way, and develop a green's-function formalism for determining the forces in the $d$-dimensional sequential packing. This picture allows one to decouple the geometric features of the packing from the pattern of transmitted forces. In Section IV we also explore the macroscopic consequences of these force laws, leading to an expression for the stress tensor with $d$ variables rather than the $d(d+1) / 2$ variables of a general stress tensor. In two dimensions, our formalism places a constraint on the stress, whose form agrees with the null stress law of Wittmer et al. [6]. In higher dimensions, the constraints on the stress also lead to a unidirectional equation for the transmission of stress in the form of a wave equation. In Section 6 we consider the relevance of our findings for real granular piles, which are not sequentially packed and which have friction. Friction can alter the transmission of forces qualitatively, restoring the elastic transmission of stress.

\section{UNDERDETERMINATION WITHIN A FRICTIONLESS PACK}

In this section we consider how the constraints inherent in the packing of impenetrable, frictionless beads determine the contact forces between the beads. Forces in frictionless packs have been studied by simulation [7, 8, 10,9]. Theoretical properties of the forces have been established for simplified systems [11 13. We begin with a summary of the well-recognized enumeration of equations and unknowns, as discussed, eg. by Alexander [22] and by the Cambridge group 11]. We then consider the role of external forces acting on a subregion of the pack, and show that a subset of these suffices to determine the others.

For definiteness we consider a system of rigid spherical beads whose sizes are chosen randomly from some continuous distribution. We suppose that the diameters of all the spheres are specified and the topology of their packing is given. That is, all the pairs of contacting beads are specified. The topology can be characterized by the average coordination number, i.e., the average number of nearest neighbors, $\bar{Z}=2 N_{c} / M$ (here $N_{c}$ is the total number of contacts, and $M$ is the number of beads). The necessary condition for the packing of a given topology to be realizable in a space of dimensionality $d$ is that the coordinates of the bead centers, $\mathbf{x}_{\alpha}$ satisfy the following equations, one for each pair of contacting beads $\alpha$ and $\beta$ :

$$
\left(\mathbf{x}_{\alpha}-\mathbf{x}_{\beta}\right)^{2}=\left(R_{\alpha}+R_{\beta}\right)^{2}
$$

Here $R_{\alpha}, R_{\beta}$ are the radii of the beads. There are $N_{c}$ such equations (one for each contact), and $M d$ variables ( $d$ for each bead). The number of equations should not exceed the number of variables; otherwise, the co-ordinates $\mathbf{x}_{\alpha}$ are overdetermined. Thus, the coordination number of a geometrically-realizable packing should not exceed the critical value of $2 d: \bar{Z} \leq 2 d$. We assume all the equations imposed by the topological constraints to be independent. If they were not independent, they would become so upon infinitesimal variation of the bead sizes. For instance, the hexagonal packing in two dimensions has the coordination number 6 which is higher then the critical value, $\langle Z\rangle=4$; but the extra contacts are eliminated by an infinitesimal variation of the bead diameters. In other words, the creation of a contact network with coordination number higher than $2 d$ occurs with probability zero in an ensemble of spheres with a continuous distribution of diameters. We shall ignore such zero-measure situations henceforth.

The above consideration gives the upper limit on the average coordination number, $\bar{Z}$. The lower limit can be 
obtained from the analysis of mechanical stability of the packing: it gives a complementary inequality: $\bar{Z} \geq 2 d$. We will consider a packing to be mechanically stable if there is a non-zero measure set of external forces which can be balanced by inter-bead ones. The packing of frictionless spheres is always characterized by $\langle Z\rangle=2 d$, as we now show. Stability requires that the net force on each bead be zero; there are $M d$ such equations. The forces in these $M d$ equations are the $N_{c}$ contact forces. The $M d$ equilibrium conditions determine the magnitudes of the $N_{c}$ contact forces. (Their directions are determined by the geometry of the packing.) The number of equilibrium equations $M d$ should not exceed the number of force variables $N_{c}$; otherwise these forces would be overdetermined. Thus $M d \leq N_{c}$, or $\bar{Z} \geq 2 d$. To avoid both overdetermined co-ordinates and overdetermined forces, we must thus have $\bar{Z}=2 d$.

Similar counting arguments have been discussed previously 22,12]. A subset of them have been applied to granular packs with friction [11]. Here we emphasize a further feature of a frictionless bead pack that has not been well appreciated: the co-ordinates and forces within a subregion of a large bead pack are necessarily underdetermined. Quantifying this indeterminacy will play an important role in our reasoning below. To exhibit the indeterminacy, we consider some compact region within the packing, containing $M^{\prime}$ beads. This unbiased selection of beads must have the same average co-ordination number $\bar{Z}$ as the system as a whole: $\bar{Z}^{\prime}=2 d$. Let $N_{\text {ext }}$ be the number of contacts of this sub-system with external beads, and $N_{\text {int }}$ be the number of the internal contacts. The average coordination number $\bar{Z}^{\prime}$ can be expressed $\bar{Z}^{\prime}=\left(N_{\text {ext }}+2 N_{\text {int }}\right) / M^{\prime}$ (any internal contact should be counted twice). Since there are $M^{\prime} d$ equations of force balance for these beads, one is able to determine all $N_{\text {ext }}+N_{\text {int }}$ contact forces in the system, whenever $M^{\prime} d=N_{\text {ext }}+N_{\text {int }}$. Evidently, if the forces on the $N_{\text {ext }}$ contacts are not specified, the internal forces cannot be computed: the system is underdetermined. The number of external forces $N_{0}$ required is given by $N_{0}=M^{\prime} d-N_{\text {int }}$. This $N_{0}$ may be related to the average co-ordination number $\bar{Z}^{\prime}$ :

$$
N_{0}=M^{\prime}\left[d-\frac{\bar{Z}^{\prime}}{2}\right]+\frac{N_{\text {ext }}}{2}
$$

We now observe that the quantity in [...] vanishes on average. This is because the average of $\bar{Z}^{\prime}$ for any subset of particles is the same as the overall average. There is no systematic change of $\bar{Z}^{\prime}$ with $M^{\prime}$. Thus if one half (on average) of mutually-independent external forces is known (let us call them "incoming" ones), the analysis of force balance in the region enables one to determine all the remaining forces, including the other half of external ones ("outgoing"). We are free to choose the incoming contacts at will, provided these give independent constraint equations.
This observation supports the unidirectional, propagating stress picture, discussed in the Introduction. Indeed, one can apply the above arguments to the slabs of the packing created by cutting it with horizontal surfaces. In a given slab of material, we choose the forces from the slab above as the incoming forces. According to the preceding argument, these should determine the outgoing forces transmitted to the slab beneath. This must be true provided that the constraints from the upper slab are independent.

Such force transmission contrasts with that of a solid body, as emphasized in the Introduction. If a given set of forces is applied to the top of a slab of elastic solid, the forces on the bottom are free to vary, provided the total force and torque on the slab are zero. Yet in our bead pack, which appears equally solid, we have just concluded that stability conditions determine all the bottom forces individually. In deducing this peculiar behavior, we did not exclude tensile forces; we may replace all the contacts by stiff springs that can exert strong positive or negative force, without altering our reasoning. In this sense our result is different from the recent similar result of Moukarzel [12]. The origin of the peculiar behavior lies in the minimal connectivity of the beads.

In a subregion of the minimal network, the constraints can be satisfied with no internal forces. Moreover, numerous (roughly $N_{\text {ext }} / 2$ ) small external displacements can be applied to the subregion without generating proportional restoring forces. We call these motions with no restoring force "soft modes". If we replace these external displacements with external forces and require no motion, compensating forces must be applied elsewhere to prevent motion of the soft modes. If the applied forces perturb all the soft modes, there must be one compensating force for each applied force to prevent them from moving - on average $N_{\text {ext }} / 2$ of them. The subregion is "transparent" to external forces, allowing them to propagate individually through the region.

This transparent behavior would be lost if further springs were added to the minimal network, increasing $\bar{Z}$. Then the forces on a subregion would be determined even without external contacts. The addition of external displacements would deform the springs, and produce proportional restoring forces. There would be no soft modes, and no transparency to external forces.

A simple square lattice of springs provides a concrete example of the multiple soft modes predicted above. Its elastic energy has the form

$$
H=K \int \mathrm{d} x \mathrm{~d} y\left[\left(u^{x x}\right)^{2}+\left(u^{y y}\right)^{2}\right]
$$

This functional does not depend on $u^{x y}$, thus there are shear deformations $\left(u^{x x}=u^{y y}=0\right)$ which cost no elastic energy. This means that the stress field should be orthogonal to any such mode, i.e.,

$$
\sigma^{i j} u_{o}^{i j}=0
$$


where $u_{o}^{x x}=u_{o}^{y y}=0$, and $u_{o}^{x y}$ is an arbitrary function of $(x ; y)$. The above equation implies that $\sigma^{x y}=0$, i.e., the principal axes of the stress tensor are fixed and directed along $x$ and $y$. This provides a necessary closure for the standard macroscopic equation of force balance,

$$
\partial^{i} \sigma^{i j}=f_{\mathrm{ext}}^{j}
$$

here $\mathbf{f}_{\text {ext }}$ is an external force. Since $\sigma^{x y}=0$ the two unknown components of the stress field, $\sigma^{x x}$ and $\sigma^{y y}$ propagate independently along the corresponding characteristics, $x=$ const and $y=$ const:

$$
\begin{aligned}
& \partial^{x} \sigma^{x x}=f_{\mathrm{ext}}^{x} \\
& \partial^{y} \sigma^{y y}=f_{\mathrm{ext}}^{y}
\end{aligned}
$$

The propagation of the solution along characteristics is a property of hyperbolic problems such as wave equation. The above equations without external force imply that each component of the stress tensor $\hat{\sigma}$ satisfies a wave equation of the form

$$
\left(\frac{\partial^{2}}{\partial t^{2}}-\frac{\partial^{2}}{\partial s^{2}}\right) \hat{\sigma}=0
$$

where $t \equiv x+y$ and $s \equiv x-y$. Thus, the fact that the original elastic energy has the soft modes results in hyperbolic, rather than elliptic equations for the stress field. One has now to specify the surface forces (or displacements) at a single non-characteristic surface - a line not parallel to $x$ or $y$ - in order to determine the stress field in all the sample.

A frictionless granular packing behaves like this example: they both are minimally coupled; they both have soft modes; they both have unidirectional propagation. In both examples only the surface of the sample stabilizes the soft modes. The above consideration of regular lattice can be easily extended to the case of arbitrary angle between the characteristic directions, $x$ and $y$. Instead of starting with a square lattice, we could have applied a uniform $x-y$ shear, altering the angle between the horizontal and vertical springs. The reasoning above holds for this lattice just as for the original square one.

The nature of the soft modes in a disordered bead pack is less obvious than in this lattice example. We have not proven, for instance, that all the forces acting on the top of a slab correspond to independent soft modes, which determine the forces at the bottom. Otherwise stated, we have not shown that the soft modes seen in the microscopic displacements have continuum counterparts in the displacement field of the region. However, the following construction, like the lattice example above, suggests that the soft modes survive in the continuum limit

To construct the pack, we place circular disks one at a time into a two-dimensional vertical channel of width $L$. (Such sequential packings will figure prominently in the next section.) Since the disks are of different sizes, the packing will be disordered. We place each successive disk at the lowest available point until the packed disks have reached a height of order $L$, as shown in Figure 2. We now construct a second packing, starting from a channel of slightly greater width $L+\delta$. We reproduce the packing constructed in the first channel as far as possible. We use an identical sequence of disks and place each at the lowest point, as before. There must be a nonvanishing range of $\delta$ for which the contact topology is identical. The motion of the wall over this range is thus a soft mode. As the side wall moves, the top surface will move by some amount $\epsilon$, proportional to $\delta$. Now, instead of holding the side wall fixed, we exert a given force $f^{x}$ on it. Likewise, we place a lid on the top, remove gravity, and exert another force $f^{y}$. Evidently unless $f^{x} / f^{y}=\epsilon / \delta$, a motion of the soft mode would result in work, and the system would move. Thus $f^{y}$ plus the condition of no motion determines $f^{x}$. This condition translates into an imposed proportionality between the stresses $\sigma^{y y}$ and $\sigma^{x x}$, as in the lattice example above. The soft modes have continuum consequences.

\section{SEQUENTIAL PACKING UNDER GRAVITY}

In the previous section we have shown that a packing of frictionless spherical beads is an anomalous solid from the point of view of classical elastic theory. The fact that the average coordination number in such a packing is exactly $2 d$ for the infinite system supports unidirectional, propagating stress. Now we elaborate this concept in more detail, by deriving particular laws for microscopic and macroscopic force transfer adopting a particular packing procedure. We suppose that the beads are deposited one by one in the presence of gravity. The weight of any new sphere added to the existing packing must be balanced by the reactions of the supporting beads. This is possible only if the number of such supporting contacts is equal to the dimensionality $d$. Any larger number of contacts requires a specific relationship between the sizes and coordinates of the supporting beads, and and thus occurs with vanishing probability. As a result, the eventual packing has an average coordination number $2 d$, like any stable, frictionless pack. In addition, it has a further property: a partial time-like ordering. Namely, among any two contacting beads there is always one which has found its place earlier than the other (the supporting one), and any bead has exactly $d$ such supporting neighbors. Note that the supporting bead is not necessarily situated below the supported one in the geometrical sense. The discussed ordering is topological rather than spatial.

One could expect that although any bead has exactly $d$ supporters at the moment of deposition, this may change later. Specifically, adding an extra bead to the packing may result in the violation of positivity of some contact force in the bulk [14]. This will lead to a rearrange- 
ment of the network. For the moment we assume that the topology of the sequential packing is preserved in the final state of the system, and return to the effect of rearrangements in Section V.

The partial ordering of the sequential packing considerably simplifies the calculation of the force distribution. Indeed, any force applied to a bead can be uniquely decomposed into the $d$ forces on the supporting contacts. This means that the force balance enables us to determine all the "outcoming" (downward) forces if the "incoming" ones are known. Therefore, there is a simple unidirectional procedure of determination of all the forces in the system. Below, we use this observation to construct a theory of stress propagation on the macroscopic scale.

\section{MEAN-FIELD STRESS}

We will characterize any inter-bead contact in a sequential packing with a unit vector directed from the center of supported bead $\alpha$ toward the supporting one $\beta$,

$$
\mathbf{n}_{\alpha \beta}=\frac{\mathbf{x}_{\beta}-\mathbf{x}_{\alpha}}{\left|\mathbf{x}_{\beta}-\mathbf{x}_{\alpha}\right|}
$$

The stress distribution in the frictionless packing is given if a non-negative magnitude of the force acting along any of the above contact unit vector is specified. We denote such scalar contact force as $f_{\alpha \beta}$

The total force to be transmitted from some bead $\alpha$ to its supporting neighbors is the sum of all the incoming and external (e.g. gravitational) forces:

$$
\mathbf{F}_{\alpha}=\left(\mathbf{f}_{\mathrm{ext}}\right)_{\alpha}+\sum_{\beta(\rightarrow \alpha)} \mathbf{n}_{\beta \alpha} f_{\beta \alpha}
$$

Here $\beta(\rightarrow \alpha)$ denotes all the beads supported by $\alpha$. Since there are exactly $d$ supporting contacts for any bead in a sequential packing, the above force can be uniquely decomposed onto the corresponding $d$ components, directed along the outcoming vectors $\mathbf{n}_{\alpha \gamma}$. This gives the values of the outcoming forces. The $f$ 's may be compactly expressed in terms of a generalized scalar product $\langle\ldots \mid \ldots\rangle_{\alpha}$ :

$$
f_{\alpha \gamma}=\left\langle\mathbf{F}_{\alpha} \mid \mathbf{n}_{\alpha \gamma}\right\rangle_{\alpha}
$$

The scalar product $\langle\ldots \mid \ldots\rangle_{\alpha}$ is defined such that $\left\langle\mathbf{n}_{\alpha \gamma} \mid \mathbf{n}_{\alpha \gamma^{\prime}}\right\rangle_{\alpha}=\delta_{\gamma \gamma^{\prime}}$. (all the Greek indices count beads, not spatial dimensions). In general, it does not coincide with the conventional scalar product. If now some force is applied to certain bead in the packing, the above projective procedure allows one to determine the response of the system, i.e. the change of the contact forces between all the beads below the given one. In other words one can follow how the perturbation propagates downward. Since the equations of mechanical equilibrium are linear, and beads are assumed to be rigid enough to preserve their sizes, the response of the system to the applied force is also linear. This linearity can be destroyed only by violating the condition of positivity of the contact forces, which implies the rearrangement of the packing. While the topology (and geometry) of the network is preserved, one can introduce the Green function to describe the response of the system to the applied forces. Namely, force $\mathbf{f}_{\lambda}$ applied to certain bead $\lambda$ results in the following additional force acting on another bead, $\mu$ (lying below $\lambda$ ):

$$
\mathbf{f}_{\mu}=\widehat{\mathbf{G}}_{\mu \lambda} \cdot \mathbf{f}_{\lambda}
$$

Here $\widehat{\mathbf{G}}_{\lambda \mu}$ is a tensor Green function, which can be calculated as the superposition of all the projection sequences (i.e. trajectories), which lead from $\lambda$ to $\mu$.

The stress field $\sigma^{i j}$ in the system of frictionless spherical beads can be introduced in the following way [27]:

$$
\sigma^{i j}(\mathbf{x})=\sum_{\alpha} \sum_{\beta(\leftarrow \alpha)} f_{\alpha \beta} n_{\alpha \beta}^{i} n_{\alpha \beta}^{j} R_{\alpha \beta} \delta\left(\mathbf{x}_{\alpha}-\mathbf{x}\right)
$$

Here $R_{\alpha \beta}=\left|\mathbf{x}_{\alpha}-\mathbf{x}_{\beta}\right|$. As we have just shown, the magnitude of the force $f_{\alpha \beta}$ transmitted along the contact unit vector $\mathbf{n}_{\alpha \beta}$ can be expressed as an appropriate projection of the total force $F_{\alpha}$ acting on the bead $\alpha$ from above. This allows one to express the stress tensor in terms of the vector field $F_{\alpha}$ :

$$
\sigma^{i j}(\mathbf{x})=\sum_{\alpha} \sum_{\beta(\leftarrow \alpha)}\left\langle F_{\alpha} \mid \mathbf{n}_{\alpha \beta}\right\rangle_{\alpha} n_{\alpha \beta}^{i} n_{\alpha \beta}^{j} R_{\alpha \beta} \delta\left(\mathbf{x}_{\alpha}-\mathbf{x}\right)
$$

In order to obtain the continuous macroscopic description of the system, one has to perform the averaging of the stress field over a region much larger than a bead. At this stage we make a mean-field approximation for the force $\mathbf{F}_{\alpha}$ acting on a given bead from above: we replace $\mathbf{F}_{\alpha}$ by its average $\overline{\mathbf{F}}$ over the region. To be valid, this assumption requires that

$$
\begin{array}{r}
\sum_{\alpha \beta}\left\langle\left(\mathbf{F}_{\alpha}-\overline{\mathbf{F}}\right) \mid \mathbf{n}_{\alpha \beta}\right\rangle_{\alpha} \mathbf{n}_{\alpha \beta}^{i} \mathbf{n}_{\alpha \beta}^{j} R_{\alpha \beta} \\
\ll \sum_{\alpha \beta}\left\langle\overline{\mathbf{F}} \mid \mathbf{n}_{\alpha \beta}\right\rangle_{\alpha} \mathbf{n}_{\alpha \beta}^{i} \mathbf{n}_{\alpha \beta}^{j} R_{\alpha \beta}
\end{array}
$$

For certain simple geometries, the mean-field approximation is exact. One example is the simple square lattice treated in Section III. In any regular lattice with one bead per unit cell, all the $\mathbf{F}_{\alpha}$ 's must be equal under any uniform applied stress. Thus replacing $\mathbf{F}_{\alpha}$ by its average changes nothing. If this lattice is distorted by displacing its soft modes, the $\mathbf{F}_{\alpha}$ are no longer equal and the validity of the mean-field approximation can be tested. Figure 3 shows a periodic distortion with fourbeads per unit cell. For example, under an applied vertical force, the bottom forces oscillate to the left and right. Nevertheless, the stress crossing the bottom row, like that crossing the row above it, is the average force times the length. One may 
verify that the $\mathbf{F}_{\alpha}$ may also be replaced by its average when the applied force is horizontal. Though the meanfield approximation is exact in these cases, it is clearly not exact in all. In the lattice of Figure 3 the mean field approximation may be inexact if one considers a region not equal to a whole number of unit cells.

A disordered packing may be viewed as a superposition of periodic soft modes like those of Figure 3. Each such mode produces fluctuating forces, like those of the example. But after averaging over an integer number of unit cells, the stress may depend on only the average force $\overline{\mathbf{F}}$. A disordered packing need not have a fixed co-ordination number as our example does. This is another possible source of departure from the mean-field result.

Now, it becomes an easy task to perform a local averaging of the Eq. 14 for the stress field in the vicinity of a given point $\mathbf{x}$, replacing $\mathbf{F}_{\alpha}$ by its average:

$$
\overline{\sigma^{i j}}(\mathbf{x})=\rho \overline{F^{k}}(\mathbf{x}) \tau^{k i j}(\mathbf{x})
$$

Here $\rho$ is the bead density, $\overline{\mathbf{F}}(\mathbf{x})$ is the force $\mathbf{F}_{\alpha}$ averaged over the beads $\alpha$ in the vicinity of the point $\mathbf{x}$, and the third-order tensor $\hat{\tau}$ characterizes the local geometry of the packing:

$$
\tau^{k i j}(\mathbf{x})=\overline{\left|\mathbf{n}_{\alpha \beta}\right\rangle_{\alpha}^{k} n_{\alpha \beta}^{i} n_{\alpha \beta}^{j} R_{\alpha \beta}}
$$

This equation is similar in spirit to one derived by Edwards for the case of a $d+1$ co-ordinated packing of spheres with friction [23]. Our geometric tensor $\tau$ plays a role analogous to that of the fabric tensor in that treatment.

The stress field satisfies the force balance equation, Eq. (5). Since this is a vector equation, it normally fails to give a complete description of the tensor stress field. In our case, however, the stress field has been expressed in terms of the vector field $\mathbf{F}$. This creates a necessary closure for the force balance equation. It is important to note that the proposed macroscopic formalism is complete for a system of arbitrary dimensionality: there is a single vector equation and a single vector variable. We now discuss the application of the above macroscopic formalism in two special cases. First we consider the equations of stress propagation in two dimensions. Then we discuss a packing of arbitrary dimensionality but with uniaxial symmetry. It is assumed to have no preferred direction other than that of gravity.

\section{A. Two-dimensional packing.}

In two dimensions, according to Eq. (16), the stress tensor $\hat{\sigma}$ can be written as a linear combination of two $\tau$ tensors.

$$
\hat{\sigma}=F_{1} \hat{\sigma}_{1}+F_{2} \hat{\sigma}_{2},
$$

where $\left[\hat{\sigma}_{1}\right]^{i j}=\tau^{1 i j}$ and $\left[\hat{\sigma}_{2}\right]^{i j}=\tau^{2 i j}$. Since the $\hat{\sigma}_{1}$ and $\hat{\sigma}_{2}$ are properties of the medium and are presumed known, the problem of finding the stress profile $\hat{\sigma}(x)$ becomes that of finding $F_{1}$ and $F_{2}$ under a given external load. Rather than determining these $F$ 's directly, we may view Eq. (18) as a constraint on $\hat{\sigma}$. The form (18) constrains $\hat{\sigma}$ to lie in a subspace of the three-dimensional space of stress components $\vec{\sigma} \equiv\left(\sigma^{x x}, \sigma^{y y}, \sigma^{x y}\right)$. It must lie in the two-dimensional subspace spanned by $\vec{\sigma}_{1}$ and $\vec{\sigma}_{2}$. This constraint amounts to one linear constraint on the components of $\sigma$, of the form

$$
\sigma^{i j} u^{i j}=0
$$

where the $\hat{u}$ tensor is determined by $\hat{\sigma}_{1}$, and $\hat{\sigma}_{2}$. Specifically, $\hat{u}$ may be found by observing that the determinant of the vectors $\vec{\sigma}, \vec{\sigma}_{1}, \vec{\sigma}_{2}$ must vanish. Expanding the determinant by minors to obtain the coefficients of the $\sigma^{i j}$, one finds

$$
\hat{u}=\left(\begin{array}{cc}
\left|\begin{array}{cc}
\sigma_{1}^{y y} & \sigma_{2}^{y y} \\
\sigma_{1}^{x y} & \sigma_{2}^{x y}
\end{array}\right|\left|\begin{array}{cc}
\sigma_{1}^{x x} & \sigma_{2}^{x x} \\
\sigma_{1}^{y y} & \sigma_{2}^{y y}
\end{array}\right| \\
\left|\begin{array}{cc}
\sigma_{1}^{x x} & \sigma_{2}^{x x} \\
\sigma_{1}^{y y} & \sigma_{2}^{y y}
\end{array}\right|\left|\begin{array}{cc}
\sigma_{1}^{x y} & \sigma_{2}^{x y} \\
\sigma_{1}^{x x} & \sigma_{2}^{x x}
\end{array}\right|
\end{array}\right)
$$

Eq. 19 has the same "null-stress" form as that introduced by Wittmer et al [6], whose original arguments were based on a qualitative analysis of the problem. By an appropriate choice of the local co-ordinates $(\xi, \eta)$, the $\hat{u}$ tensor can be transformed into co-ordinates such that $u^{\xi \xi}=u^{\eta \eta}=0$. Then the null stress condition becomes $\sigma^{\xi \eta}=\sigma^{\eta \xi}=0$. This implies that, according to force balance equation (5), the non-zero diagonal components of the stress tensor "propagate" independently along the corresponding characteristics, $\xi=$ const and $\eta=$ const:

$$
\partial^{\xi} \sigma^{\xi \xi}=f_{\text {ext }}^{\xi} \quad \partial^{\eta} \sigma^{\eta \eta}=f_{\text {ext }}^{\eta}
$$

Our microscopic approach gives an alternative foundation for the null-stress condition, Eq. (19), and allows one to relate the tensor $\hat{u}$ in this equation to the local geometry of the packing. Our general formalism is not limited to the two-dimensional case, and in this sense, is a generalization of the null-stress approach.

\section{B. Axially-symmetric packing.}

Generally, there are two preferred directions in the sequential packing: that of the gravitational force, $\mathbf{g}$, and that of the growth surface $\mathbf{n}$. In the case when these two directions coincide, the form of the third-order tensor $\hat{\tau}$, Eq. (17), should be consistent with the axial symmetry associated with the single preferred direction, $\mathbf{n}$. Since $\tau^{k i j}$ is symmetric with respect to $i \leftrightarrow j$ permutation, it can be only a linear combination of three tensors: $n^{k} n^{i} n^{j}$, $n^{k} \delta^{i j}$ and $\delta^{k i} n^{j}+\delta^{k j} n^{i}$, for general spatial dimension $d$.

Let $\sigma^{i j}$ be the stress tensor in the $d$-dimensional space $(i, j=0 \ldots d-1$, and index ' 0 ' corresponds to the vertical direction). From the point of view of rotation around 
the vertical axis the stress splits into scalar $\sigma^{00}, d-1$ dimensional vector $\sigma^{0 a}(a=1 \ldots d-1) d-1$ dimensional tensor $\sigma^{a b}$. According to our constitutive equation 16, the stress should be linear in vector $\mathbf{F}$, which itself splits into a scalar $F^{0}$ and a vector $F^{a}$ with respect to horizontal rotations. Since the material tensor $\tau$ is by hypothesis axially symmetric, the only way that the "scalar" $\sigma^{00}$ may depend on $\mathbf{F}$ is to be proportional to "scalar" $F^{0}$. Likewise, the only way "tensor" $\sigma^{a b}$ can be linear in $\mathbf{F}$ is to be proportional to $\delta^{a b} F^{0}$. Therefore, in the axially-symmetric case

$$
\sigma^{a b}=\lambda \delta^{a b} \sigma^{00}
$$

where the constant $\lambda$ is eg. $\tau^{011} / \tau^{000}$. This constitutive equation allows one to convert the force balance equation (5) to the following form:

$$
\partial^{0} \sigma^{00}+\partial^{a} \sigma^{a 0}=f_{e x t}^{0} ; \quad \partial^{0} \sigma^{a 0}+\lambda \partial^{a} \sigma^{00}=f_{e x t}^{a}
$$

In the case of no external force, we may take $\partial^{0}$ of the first equation and combine with the second to yield a wave equation for $\sigma^{00}$. Evidently $\sigma^{a b}$, being a fixed multiple of $\sigma^{00}$, obeys the same equation. Similar manipulation yields the same wave equation for $\sigma^{0 a}$ and $\sigma^{a 0}$. Thus every component of stress satisfies the wave equation with vertical direction playing the role of time and $\sqrt{\lambda}$ being the propagation velocity.

\section{DISCUSSION}

In this section we consider how well our model should describe real systems of rigid, packed units. As stated above, our model is most relevant for emulsions or dense colloidal suspensions, whose elementary units ae well described as frictionless spheres. Under very weak compression the forces between such units match our model assumptions. However, our artificial procedure of sequential packing bears no obvious resemblance to the arrangements in real suspensions. We argue below that our model may well have value even when the packing is not sequential. More broadly we may consider the connection between our frictionless model and real granular materials with friction. The qualitative effect of adding friction to our sequential packing is to add constraints so that the network of contacts is no longer minimally connected. Thus the basis for a null-stress description of the force transmission is compromised. We argue below that friction should cause forces to propagate as in an elastic medium, not via null-stress behavior.

\section{A. Sequential packing}

We first consider the consequences of our sequential packing assumption. One consequence is that each bead has exactly $d$ supporting contacts. These lead successively to earlier particles, forming a treelike connectivity from supported beads to supporters. Although the counting arguments of Section II show that the propagating stress approach should be applicable to a wide class of frictionless systems, the continuum description of Section IV depends strongly on the assumed sequential order. Now, most packings are not sequential, and even when beads are deposited in sequence, they may undergo rearrangements that alter the network of connections. However, it is possible to modify our arguments to take account of such re-arrangements. Our reasoning depends on the existence of $d$ supporting contacts for each bead. Further, every sequence of supporting contacts starting at a given bead must reach the boundary of the system without passing through the starting bead: there must be no closed loops in the sequence.

Even in a non-sequential packing we may define a network of supporting contacts. First we define a downward direction. Then, for any given bead in the pack, we define the supporting contacts to be the $d$ lowest contacts. With probability 1 , each bead has at least $d$ contacts. Otherwise it is not stable. Typically a supporting bead lies lower than the given bead. Thus the typical sequence of supporting contacts leads predominantly downward, away from the given bead, and returns only rarely to the original height. A return to the original bead must be even more rare. One may estimate the probability that there is a loop path of supporting contacts under simple assumptions about the packing. As an example we suppose the contacts on a given bead to be randomly distributed amongst the 12 sites of a randomly-oriented close-packed lattice. We further imagine that these sites are chosen independently for each bead, with at least one below the horizontal. Then the paths are biased random walks with a mean steplength of .51 diameters and a root-mean-square steplength of about 1.2 times the mean. The probability of a net upward displacement of 1 or more diameter is about one percent. It appears that our neglect of loop paths is not unreasonable.

\section{B. Friction}

The introduction of friction strongly affects most of our arguments. Friction creates transverse as well as normal forces at the contacts. The problem is to determine positions and orientations of the beads that lead to balanced forces and torques on each. If the contact network is minimally connected, the forces can be determined without reference to deformations of the particle. But if the network has additional constraints, it is impossible to satisfy these without considering deformation. This is no less true if the beads are presumed very rigid. We first give an example to show that in a generic packing the deformability alters the force distribution substantially. We then give a prescription for defining the deformation 
and hence the contact forces unambiguously.

In our example we imagine a two-dimensional sequential packing and focus on a particular bead, labeled 0 , as pictured in Figure 1 . We presume that the beads are deposited gently, so that each contact forms without tangential force. Thus when the bead is deposited, it is minimally connected: its weight determines the two (normal) supporting forces, labeled 1 and 2. Thenceforth no slipping is allowed at the contact. Later during the construction of the pack bead 0 must support the force from some subsequent bead. This new force is normal, since it too arises from an initial contact. But the new force creates tangential forces on the supporting contacts 1 and 2. To gauge their magnitude, we first suppose that there is no friction at contacts 1 and 2, while the supporting beads remain immobile. Then the added force $F$ leads to a compression. We denote the compression of the contact 1 as $\delta$. With no friction, the contact 2 would undergo a slipping displacement by an amount of order $\delta$. Friction forbids this slipping and decrees deformation of the contact instead. The original displacement there would create an elastic restoring force of the same order as the original $F$. Thus the imposition of friction creates new forces whose strength is comparable to those without friction. The frictional forces are not negligible, even if the beads are rigid. Increasing the rigidity lessens the displacements $\delta$ associated with the given force $F$, but it does not alter the ratio of frictional to normal forces. Neither are the frictional forces large compared to the normal forces. Thus a coefficient of friction $\mu$ of order unity should be sufficient to generate enough frictional force to prevent slipping of a substantial fraction of the contacts.

The contact forces $T_{1}$ and $T_{2}$ cannot be determined by force balance alone, as they could in the frictionless case. Now the actual contact forces are those which minimize the elastic energy of deformation near the two contacts. This argument holds not just for spheres but for general rounded objects.

Though the new tangential forces complicate the determination of the forces, the determination need not be ambiguous. We illustrate this point for a sequential packing on a bumpy surface with perfect friction. We choose a placement of the successive beads so that no contact re-arrangements occur. If only a few beads have been deposited in the container, the forces are clearly well determined. Further, if the forces are presumed welldetermined up to the $M$ th bead, they remain so upon addition of the $M+1$ st bead. We presume as before that the new bead exerts only normal forces on its immediate supporters. Each supporter thus experiences a well-defined force, as shown in Section [1]. But by hypothesis, these supporting beads are part of a well-connected, solid object, whose contacts may be regarded as fastened together. Thus the displacem ents and rotations of each bead are a well-defined function of any small applied load. Once the $M+1$ 'st bead has been added, its supporting contacts also support tangential force, so that it responds to future loads as part of the elastic body.

We conclude that a sequential packing with perfect friction, under conditions that prevent contact rearrangements, transmits forces like a solid body. Small changes in stress $\delta \sigma^{i j}$ in a region give rise to proportional changes in the strain $\delta \gamma^{k \ell}$. This proportionality is summarized by an elasticity tensor $K^{i j k \ell}: \delta \sigma^{i j}=K^{i j k \ell} \delta \gamma^{k \ell}$. The elastic tensor $K$ should depend in general on how the pack was formed; thus it may well be anisotropic.

This elastic picture is compromised when the limitations of friction are taken into account. As new beads are added, underlying contacts such as contacts 1 and 2 of Figure 1 may slip if the tangential force becomes too great. Each slipping contact relaxes so as to satisfy a fixed relationship between its normal force $N$ and its tangential force $T: v i z .|T|=\mu|N|$. If $\mu$ were very small, virtually all the contacts would slip until their tangential force were nearly zero. Then the amount of stress associated with the redundant constraints must become small and the corresponding elastic moduli must become weak. Moreover, as $\mu$ approaches 0 , the material on any given scale must become difficult to distinguish from a frictionless material with unidirectional stress propagation. Still, redundant constraints remain on the average and thus the ultimate behavior at large length scales (for a given $\mu$ ) must be elastic, provided the material remains homogeneous.

\section{Force-generated contacts}

Throughout the discussion of frictionless packs we have ignored geometric configurations with probability zero, such as beads with redundant contacts. Such contacts occur in a close-packed lattice of identical disks, for example. Though such configurations are arbitrarily rare in principle, they may nevertheless play a role in real bead packs. Real bead packs have a finite compressibility; compression of beads can create redundant contacts. Thus for example a close-packed lattice of identical spheres has six contacts per bead, but if there is a slight variability in size, the number of contacts drops to four. The remaining two beads adjacent to a given bead do not quite touch. These remaining beads can be made to touch again if sufficient compressive stress is applied. Such stress-induced redundant contacts must occur in a real bead with some nonzero density under any nonzero load. These extra contacts serve to stabilize the pack, removing the indeterminate forces discussed in Section II. To estimate the importance of this effect, we consider a large bead pack compressed by a small factor $\gamma$. This overall strain compresses a typical contact by a factor of order $\gamma$ as well. The number of new contacts may be inferred from the pair correlation function $g(r)$. Data on this $g(r)$ is available for some computergenerated sequential packings of identical spheres of radius $R$ [28]. These data show that $g(r)$ has a finite value 
near 1 at $r=2 R$. Thus the number of additional contacts per bead that form under a slight compression by an amount $\delta r$ is given by $\delta \bar{Z}=6 \phi g(2 R) \delta r / R \simeq 4 \gamma$. Here $\phi \simeq .6$ is the volume fraction of the beads. These extra contacts impose constraints that reduce the number of undetermined boundary forces in a compact region containing $M^{\prime}$ beads and roughly $M^{\prime 2 / 3}$ surface beads. The remaining number of undetermined boundary forces now averages $\frac{1}{2} N_{\text {ext }}-M^{\prime} \delta \bar{Z}$. The first term is of order $M^{2 / 3}$, and must thus be smaller than the second term for $M^{1 / 3} \simeq(\delta \bar{Z})^{-1}$. For $M^{\prime}$ larger than this amount, there are no further undetermined forces and the region becomes mechanically stable. Moukarzel [12] reaches a similar conclusion by a somewhat different route.

If the pack is compressed by a factor of $\gamma$, stability occurs for $M^{\prime 1 / 3} \gtrsim 1 / \gamma$ - a region roughly $1 / \gamma$ beads across. In a typical experiment 29 the contact compression $\gamma$ is $10^{-4}$ or less, and the system size is far smaller than $10^{4}$ beads. Thus compression-induced stability should be a minor effect here. Still, this compression-induced stability might well play a significant role for large and highly compressed bead packs such as compressed emulsions [8]. In some of the large packs of Ref. [3], compressioninduced stability may also be important.

\section{Experimental evidence}

We have argued above that undeformed, frictionless beads should show unidirectional, propagating forces while beads with friction should show elastic spreading of forces. The most direct test of these contrasting behaviors is to measure the response to a local perturbing force [14]. Thus eg. if the pile of Figure 11 is a nullstress medium, the local perturbing force should propagate along a light cone and should thus be concentrated in a ring-like region at the bottom [15]. By contrast, if the pile is an elastic medium the perturbing force should be spread in a broad pattern at the bottom, with a maximum directly under the applied force. Existing experimental information seems inadequate to test either prediction, but experiments to measure such responses are in progress [30].

As noted above, emulsions and colloids are good realizations of the frictionless case. The contacts in such systems are typically organized by hydrostatic pressure or by flow, rather than by gravity. Still, our general arguments for unidirectional propagation should apply. Extensive mechanical measurements of these systems have been made 26,24]. The shear modulus study of Weitz and Mason [26] illustrates the issues. The study spans the range from liquid-like behavior at low volume fractions to solid-like behavior at high volume fractions. In between these two regimes should lie a state where the emulsion droplets are well connected but little deformed. The emulsion in this state should show unidirectional force transmission. It is not clear how this should affect the measured apparent moduli.

Other indirect information about force propagation comes from the load distribution of a granular pack on its container, such as the celebrated central dip under a conical heap of sand [3]. These data amply show that the mechanical properties of a pack depend on how it was constructed. Theories postulating null-stress behavior have successfully explained these data [6]. But conventional elasto-plastic theories have also proved capable of producing a central dip [5]. An anisotropic elastic tensor may also be capable of explaining the central dip.

Another source of information is the statistical distribution of individual contact forces within the pack or at its boundaries. The measured forces become exponentially rare for strong forces [17,20]. Such exponential falloff is predicted by Coppersmith's "q model" [17], which postulates unidirectional force propagation. Still, it is not clear whether this exponential falloff is a distinguishing feature of unidirectional propagation. A disordered elastic material might well show a similar exponential distribution.

Computer simulations should also be able to test our predictions. Recent simulations 31,8 have focussed on stress-induced restructuring of the force-bearing contact network. We are not aware of a simulation study of the transmission of a local perturbing force. Such a perturbation study seems quite feasible and would be a valuable test. We have performed a simple simulation to test the mean-field description of stress in frictionless packs. Preliminary results agree well with the predictions. An account of our simulations will be published separately.

\section{CONCLUSION}

In this study we have aimed to understand how force is transmitted in granular media, whether via elastic response or via unidirectional propagation. We have identified a class of disordered systems that ought to show unidirectional propagation. Namely, we have shown that in a general case a system of frictionless rigid particles must be isostatic, or minimally connected. That is, all the inter-particle forces can in principle be determined from the force balance equations. This contrasts with statically undetermined, elastic systems, in which the forces cannot be determined without self-consistently finding the displacements induced by those forces. Our general equation-counting arguments suggest that isostatic property of the frictionless packing results in the unidirectional propagation of the macroscopic stress.

We were able to demonstrate this unidirectional propagation explicitly by specializing to the case of sequential packing. Here the stress obeys a condition of the previously postulated null-stress form [6]; our system provides a microscopic justification for the null-stress postulate. Further, we could determine the numerical coefficients entering the null-stress law from statistical averages of 
the contact angles by using a mean field hypothesis (decoupling Anzatz). We have devised a numerical simulation to test the adequacy of the sequential packing assumption and the mean-field hypothesis. The results will be reported elsewhere.

If we add friction in order to describe macroscopic granular packs more accurately, the packing of rigid particles no longer needs to be isostatic, and the system is expected to revert to elastic behavior. This elasticity does not arise from softness of the beads or from a peculiar choice of contact network. It arises because contacts that provide only minimal constraints when created can provide redundant constraints upon further loading.

We expect our formalism to be useful in understanding experimental granular systems. It is most applicable to dense colloidal suspensions, where static friction is normally negligible. Here we expect null-stress behavior to emerge at scales large enough that the suspension may be considered uniform. We further expect that our meanfield methods will be useful in estimating the coefficients in the null-stress laws. In macroscopic granular packs our formalism is less applicable because these packs have friction. Still, this friction may be small enough in many situations that our picture remains useful. Then our microscopic justification may account for the practical success of the null-stress postulate [6] for these systems.

\section{ACKNOWLEDGEMENT}

The authors are grateful to the Institute for Theoretical Physics in Santa Barbara, for hospitality and support that enabled this research to be initiated. The authors thank the participants in the Institute's program on Jamming and Rheology for many valuable discussions. Many of the ideas reported here had their roots in the floppy network theory of Shlomo Alexander. The authors dedicate this paper to his memory. This work was supported in part by the National Science Foundation under Award numbers PHY-94 07194, DMR-9528957 and DMR 9400379 .

[1] For a review, see E. Guyon, S. Roux, A. Hansen, D. Bideau, J.-P. Troadec and H. Crapo, Rep. Prog. Phys. 53373 (1990).

[2] Friction, Arching, Contact Dynamics edited by D. E. Wolf and P. Grassberger (Singapore, World Scientific, 1997).

[3] T. Jotaki and R. Moriyama, J. Soc. Powder Technol. Jpn 60, 184 (1979); J. Smid and J. Novosad in Proc. of 1981 Powtech Conf., Ind.Chem. Eng. Symp. 63, D3V 112 (1981); R. Brockbank, J. M. Huntley and R. C. Ball, J. Physique II, 7, 1521 (1997).
[4] S. F. Edwards, C. C. Mounfield Physica A 226 (1996).

[5] J. D. Goddard and A. K. Didwania Quarterly J. Mech. Appl. Math. 51 15, (1998); F. Cantelaube and J. D. Goddard Powders and Grains 97 (R. P. Behringer and J. T. Jenkins, eds (Rotterdam, Balkema 1997) p. 185

[6] J. P. Wittmer, P. Claudin, M. E. Cates and J. P. Bouchaud Nature 382336 (1996); J. P. Wittmer, M. E. Cates and P. Claudin, J. Phys. I France 7, 39 (1997).

[7] Lacasse MD, Grest GS, Levine D Phys Rev E 545436 (1996)

[8] S. A. Langer, Andrea J. Liu, J. Phys. Chem. B 101, 8667-8671 (1997)

[9] R. S. Farr, J. R. Melrose, and R. C. Ball Phys. Rev. E. 557203 (1997).

[10] S. Luding, Phys. Rev. E 554720 (1997)

[11] S. F. Edwards, Physica A 249, 226 (1998).

[12] C. F. Moukarzel cond-mat/9803120 Phys. Rev. Lett. 81 1634 (1998); proceedings of Rigidity theory and applications Traverse City MI, June 14-18 1998, Fundamental Material Science Series, Plenum

[13] G. Oron and H. J. Herrmann Phys. Rev. E 582079 (1998).

[14] P. Claudin and J. P. Bouchaud Phys. Rev. Lett. 78231 (1997); P. Claudin, J.-P. Bouchaud, M. E. Cates and J. P. Wittmer Phys. Rev. E 574441 (1998).

[15] M. E. Cates, J. P. Wittmer, J. P. Bouchaud, and P. Claudin Phys. Rev. Lett. 811841 (1998).

[16] J. N. Goodier, Phil. Mag. 22 (1936) 678; H. M. Smallwood, J. Appl. Phys. 15 (1944) 758; E. Guth, J. Appl. Phys. 16 (1945) 20; Z. Hashin, Proc. 4th Internat. Congr. Rheol vol. 3, E. H. Lee, ed., (Interscience, New York, 1965) p. 30.

[17] S. N. Coppersmith, C-H Liu, S. Majumdar,O. Narayan, T. A. Witten Phys. Rev. E. 534673 (1996)

[18] J. E. S. Socolar Phys. Rev. E 573204 (1998).

[19] E. Clément and C. Eloy and J. Rajchenbach and J. Duran, Stochastic in Lectures on stochastic dynamics, eds. T. Pöschel and L. Schimanski-Geier, (Heidelberg Springer 1997).

[20] D. M. Mueth, H. M. Jaeger and S. R. Nagel Phys. Rev. E. 573164 (1998)

[21] F. Radjai and M. Jean and J. J. Moreau and S. Roux, Phys. Rev. Lett. 77274 (1996).

[22] S. Alexander, Physics Reports 29665 (1998).

[23] R. C. Ball, private communication September, 1997; R. C. Ball and S. F. Edwards, in preparation; D. V. Grinev and R. C. Ball, in preparation.

[24] J. C. VanderWerff, C. G. Denkruif, C. Blom, and J. Mellema, Phys. Rev. A 39795 (1989).

[25] Watanabe H, Yao ML, Osaki K, Shikata T, Niwa H, Morishima Y Rheologica Acta 36 (1997); Nonlinear rheology and flow-induced structure in a concentrated spherical silica suspension, Rheol Acta 37 1-6 (1998)

[26] T. G. Mason, J. Bibette, and D. A. Weitz Phys. Rev. Lett. 752051 (1995).

[27] L. D. Landau and E. M. Lifshitz, Theory of Elasticity 3rd edition (Oxford, Pergamon 1986) Section 2.

[28] R. Jullien, A. Pavlovitch, and P. Meakin, J. Phys. A 25 4103 (1992)

[29] C-H. Liu and S. R. Nagel Phys. Rev. B. 4815646 (1993) 
[30] S. Nagel, private communication; J.-P. Bouchaud, private communication.

[31] C. Thornton and J. Lanier Powders and Grains 97 (R. P. Behringer and J. T. Jenkins, eds (Rotterdam, Balkema 1997) p. 223

[32] B. Miller, C. O'Hern and R. P. Behringer Phys. Rev. Lett. 773110 (1996)

\section{FIGURES}
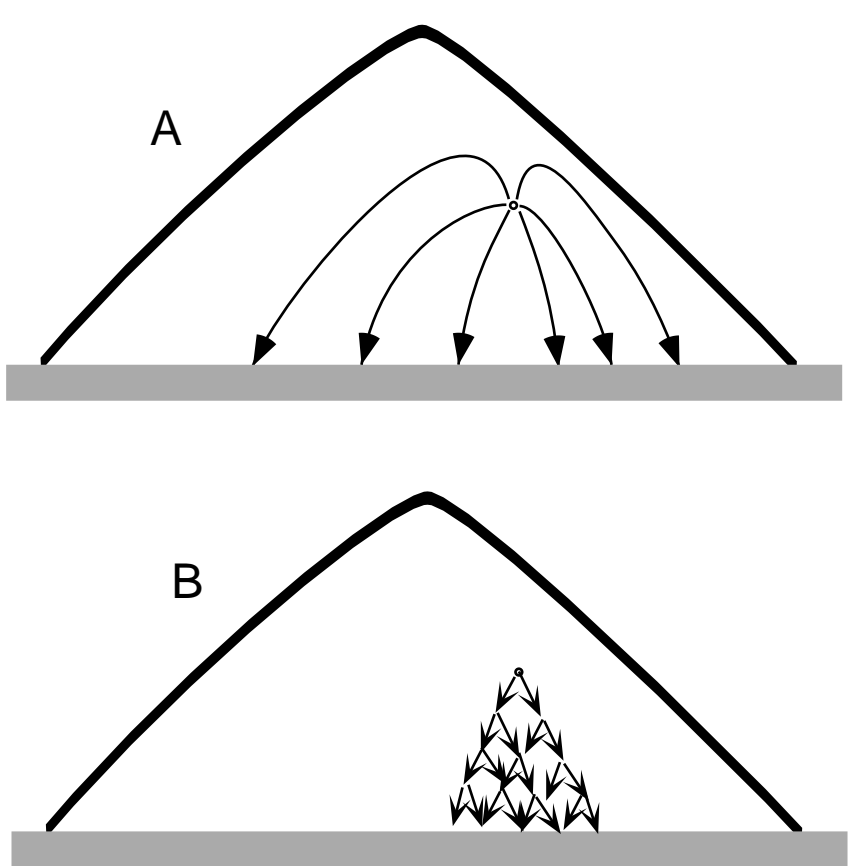

FIG. 1. Contrast between elastic (elliptical) and unidirectional (hyperbolic) force propagation in a sand-pile shaped object. A: elastic response to an imposed local downward force. Pictured lines of force represent the current of vertical force, i.e., the stress contracted into a downward unit vector. Near the source, this field is symmetric about a horizontal plane through the object 16]; part of the force is transmitted through points above the source. B: unidirectional response to an imposed local downward force. The imposed force is transmitted to neighbors below the source, and is further transmitted to neighbors below these. No force is transmitted to points above the source. 


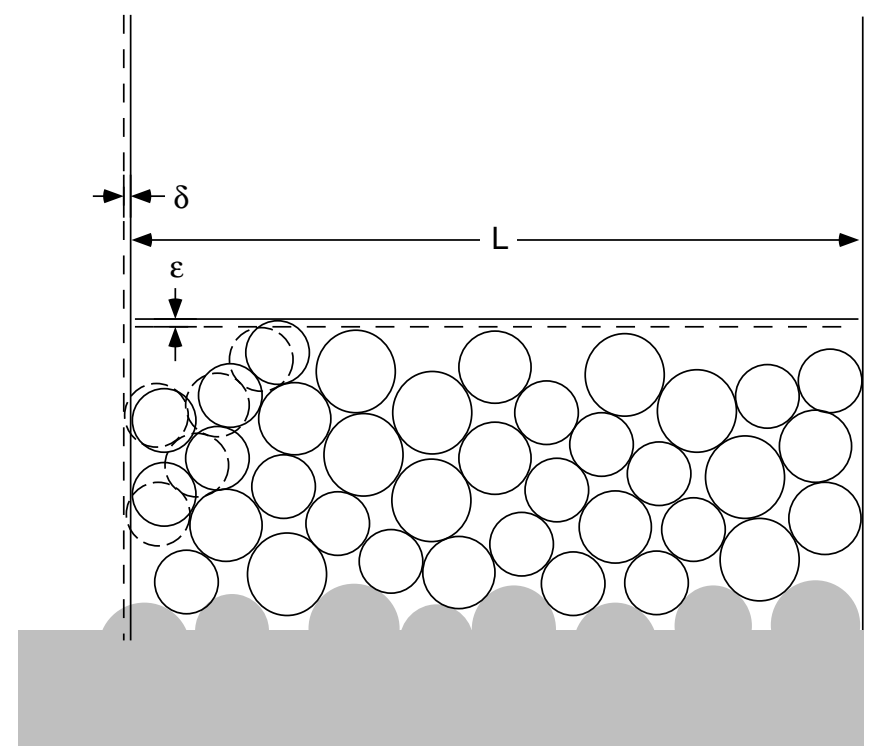

FIG. 2. A sequential packing in a channel. When left wall is displaced outward by a small amount $\delta$, the beads shift to the positions shown by dashed lines, and the top of the pile shifts by an amount $\epsilon$.



FIG. 3. A buckled square lattice illustrating the propagation of inhomogeneous forces. Bottom row of sites has alternating wide and narrow spacing. Arrows indicate the unequal forces on these sites.

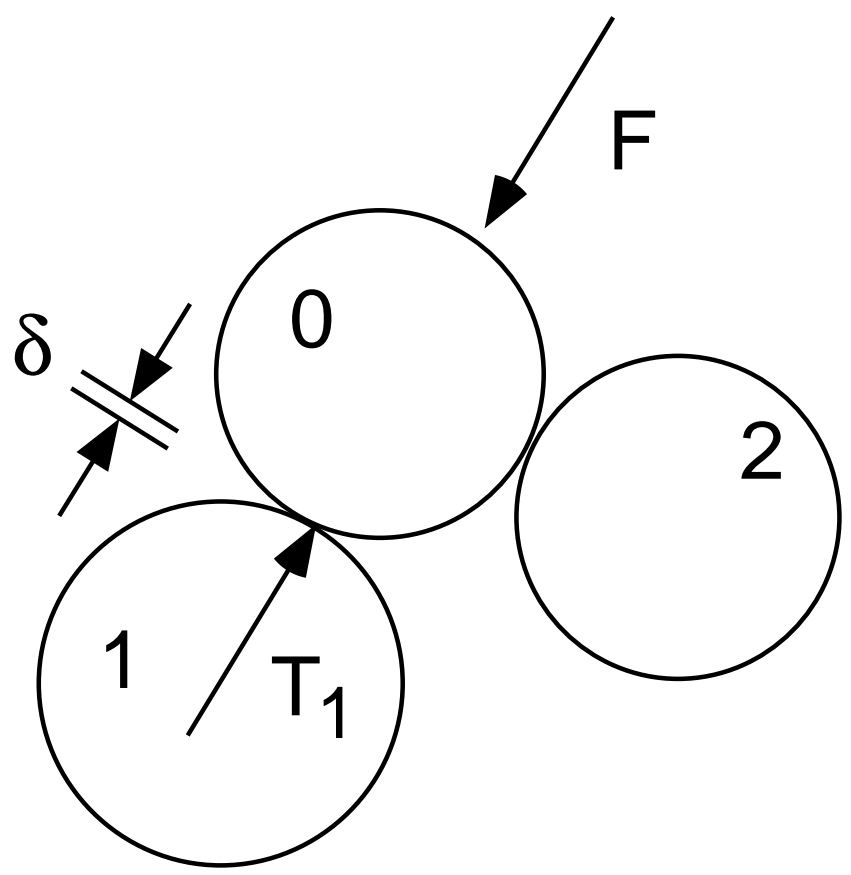

FIG. 4. The effect of friction on a triad of beads. In the absence of friction, the applied force $F$ is transmitted entirely to contact 1 , causing a displacement $\delta$. This would result in a sliding displacement of contact 2 by an amount $\delta$. With friction, contact 2 cannot slide; it must deform the contact region by an amount of order $\delta$. Thus the applied force $F$ is shared between contacts 1 and 2 . The force is distributed so as to minimize the total elastic energy at contacts 1 and 2 . 